\title{
An Optimal Automatic Beat Detection Algorithm Based on Detector Switching
}

\author{
P Tchuidjang ${ }^{1}, \mathrm{C} \mathrm{Corsi}^{1}, \mathrm{~J}^{\mathrm{De}} \mathrm{Bie}^{2}$ \\ ${ }^{1}$ DEIS, University of Bologna, Cesena, Italy \\ ${ }^{2}$ Mortara Rangoni Europe srl, Bologna, Italy
}

\begin{abstract}
In this paper we present an innovative approach to improve the performance of beat detection in the presence of a significant level of noise on some ECG leads in a setting of multi-lead acquisition. The idea is to run simultaneously two detectors ("principal" and "spare") and to dynamically switch between them according to the level of noise on the leads used by the detectors. First, default independent leads are assigned to the detectors; then, a bootstrap phase is necessary during which average QRS amplitudes are established for all leads. Based on the independence of the leads and the QRS amplitudes best leads are automatically computed and assigned to the detectors. After the bootstrap phase, the algorithm calculates the noise level of the leads used by both detectors every $500 \mathrm{~ms}$ and switches to the spare detector if the noise in the principal detector leads exceeds a threshold (T). The performance of the algorithm was tested on a database created for the purpose; the database contains 101 1-hour 12-lead Holter records selected from 24-hour recordings with a commercial analysis system on the basis of the amount of artifact. The performance of the algorithm was tested for various values of $T$ and noise observation windows. A QRS sensitivity of $99.7 \%$, a QRS positive predictive value of $99.3 \%$, a ventricular sensitivity of $91.0 \%$ and a ventricular positive predictive value of $55.3 \%$ have been achieved.
\end{abstract}

\section{Introduction}

Continuous monitoring of the electrocardiogram (ECG) in both in- and out-patients has become a very common procedure, with diverse algorithms ranging from screening for cardiac arrhythmias to surgical and critical care monitoring. As clinical experience has led to the identification of more prognostic indicators in the ECG, an increasingly number of sophisticated automated cardiac arrhythmias detection algorithm has been proposed. The early heart rate monitors rapidly evolved into devices that were designed first to detect ventricular fibrillation, then other "premonitory" ventricular arrhythmias. Many newer devices attempt to detect supraventricular arrhythmias and transient ischemic ST changes. Accurate diagnosis of ECG abnormalities requires attention to subtle features of the signals, features that may appear only rarely, and which are often obscured by or mimicked by noise. Diagnostic criteria are complicated by inter- and intra-patient variability of both normal and abnormal ECG features. Given these considerations, it is not surprising that the design of algorithms for automated ECG analysis is faced with a difficult task, and that the results of this effort are imperfect. Certain parts of the problem - QRS detection in the absence of noise, for example - are well-solved by most current algorithms; others - detection of cardiac arrhythmias, for example - remain exceedingly difficult.

In this paper, a new approach to cardiac arrhythmias automatic detection using two identical detectors is presented. The new algorithm runs simultaneously both detectors and switching among them is performed according to the electrode level of noise.

\section{Methods}

To automatically select the optimal channels detection in presence of variable level of noise we assumed that (1) most of the beat recognition errors are caused by noise, (2) noise is usually located and related to specific electrode sites and (3) intelligent switching between channels can improve the performance of the beat detection.

The method to obtain the study goal is to run simultaneously two beat detectors that work on independent channels (derived from different electrodes), and switch between them on the basis of the presence of noise. One of the detectors is called "Principal" detector, and the other is the "Spare" detector. Whenever the conditions of the Principal detector are not favorable anymore, a switch is made to the Spare detector, while the Principal now becomes the new Spare.

The structure of the algorithm implemented in the single beat detector follows the algorithmic structure developed in the early years of automated QRS detection [1] and now shared by many algorithms. 
The algorithm takes three channels as input: two of them are used for the beat detection and the third only for the QRS classification; the proposed algorithm is achieved through three steps: (1) creation of independent leads, (2) selection of suitable independent leads and (3) switch between the two detectors.

\subsection{Creation of independent leads}

The first step of the algorithm consists in creating independent bipolar leads from the original front-end channels that are all (except one) dependent on the right arm electrode. Bipolar sets are chosen on the basis of independence and probable signal strengths. Channels are marked disabled on the basis of the Lead Fail (LF) map: any electrode in fail in the map causes the channel to be disabled.

Table 1. Transformation from dependent to independent leads for a 10-wire cable. Legend: LA/RA - left/right arm; LL - left leg; C1 $\div \mathrm{C} 6$ - pre-cordial electrodes.

\begin{tabular}{|c|c|c|c|c|c|}
\hline Ch\# & $\begin{array}{c}\text { Origina } \\
\text { l signal }\end{array}$ & $\begin{array}{c}\text { New } \\
\text { Signal }\end{array}$ & $\begin{array}{c}\text { Orig } \\
\text { to New }\end{array}$ & $\begin{array}{c}\text { Orig LF } \\
\text { map }\end{array}$ & New LF map \\
\hline 0 & LA-LL & LA-LL & 0 & RA,LA,LL & RA,LA,LL \\
\hline 1 & LL-RA & LL-RA & 1 & RA,LL & RA,LL \\
\hline 2 & C1-RA & C1-LL & $2-1$ & RA, C1 & RA, LL, C1 \\
\hline 3 & C2-RA & C2-LA & $3-0-1$ & RA, C2 & RA, LA, LL, C2 \\
\hline 4 & C3-RA & C3-C6 & $4-7$ & RA, C3 & RA, C3, C6 \\
\hline 5 & C4-RA & C4-C1 & $5-2$ & RA, C4 & RA, C1, C4 \\
\hline 6 & C5-RA & C5-RA & 6 & RA, C5 & RA, C5 \\
\hline 7 & C6-RA & C6-C2 & $7-3$ & RA, C6 & RA, C2, C6 \\
\hline
\end{tabular}

\subsection{Selection of independent leads}

The goal of the second step is to select automatically the most appropriate detection channel combinations for each beat detector. This is done on the basis of QRS amplitude and electrode independence.

An initial bootstrap procedure is run to measure the QRS amplitude. The Principal detector maintains a running average of the last 32 normal beat amplitudes for each channel (0-7 for 10-wire). At first start-up, the detection channels assigned to both detectors are presented in the following table 2 .

Table 2. Detection channels assigned to detectors at first start up

\begin{tabular}{|l|c|}
\hline \multicolumn{1}{|c|}{ Channel } & 10 wire cable \\
\hline Detector A, detection channel 1 & 1 (LL-RA) \\
\hline Detector A, detection channel 2 & 4 (C3-C6) \\
\hline Detector A, confirmation channel & $3(\mathrm{C} 2-\mathrm{LA})$ \\
\hline Detector B, detection channel 1 & $3(\mathrm{C} 2-\mathrm{LA})$ \\
\hline Detector B, detection channel 2 & $5(\mathrm{C} 4-\mathrm{C} 1)$ \\
\hline Detector B, confirmation channel & $6(\mathrm{C} 5-\mathrm{RA})$ \\
\hline
\end{tabular}

At start up, detector A is the Principal detector and detector B is the Spare detector. Both detectors are started simultaneously, and must be kept in synchronization and any detector switching is suspended. Once the Principal detector has acquired the first 32 normal beats, the new detection channels are computed and a rhythm relearn procedure starts. Detection leads are determined as follows:

1. Det $1=$ the channel with the maximum amplitude

2. Det $2=$ the next channel with maximum amplitude and greater than a minimum QRS amplitude (wMinQrsAmp). If possible with no electrodes in common with Det1;

3. Det $3=$ the next channel with maximum amplitude and greater than wMinQrsAmp. If possible with no electrodes in common with Det1 and Det2;

4. Det $4=$ the next channel with maximum amplitude and greater than wMinQrsAmp. If possible with no electrodes in common with Det1, Det2 and Det3;

5. Det5= the next channel with maximum amplitude;

6. Det $6=$ the next channel with maximum amplitude;

Disabled channels are excluded, that is they are the last ones selected as detection channels. If there is only one valid channel, it will be allocated to Detector A as detection channel 1. Detector A becomes the Principal detector and detector switching is disabled until the next relearn procedure is performed. The new detection channels are assigned to both detectors as illustrated in the following table 3 .

Table 3. Detection channels assigned to detectors at first start up

\begin{tabular}{|l|c|}
\hline \multicolumn{1}{|c|}{ Detector } & Detector channel \\
\hline Detector A, detection channel 1 & Det1 \\
\hline Detector A, detection channel 2 & Det4 \\
\hline Detector A, confirmation channel & Det5 \\
\hline Detector B, detection channel 1 & Det2 \\
\hline Detector B, detection channel 2 & Det3 \\
\hline Detector B, confirmation channel & Det6 \\
\hline
\end{tabular}

Once detection channels are automatically computed and assigned to both detectors, a rhythm learn command is given to the Spare detector with the new detection channels. When relearn on this detector is completed, a switch of detectors is made, and a rhythm learn command is now given to the other detector (which has now become the new Spare), also with the new detection channels. The relearn procedure ends when also this detector finishes rhythm relearn. Detector switching remains on hold for one more minute, and then detector switching is possible according to the level of noise.

\subsection{Detector switching}

Detector switching is performed on the basis of a parameter "dNPR" and a threshold "T". dNPR represents an estmation of the level of noise in a detection channel 
and it is computed for any ECG sample. The Principal and the Spare detectors are respectively indicated by the letters "P" and "S". dNPR[0] and dNPR[1] indicate the parameter dNPR respectively for the first and the second detection channel and they are computed for each detector; thus $\mathrm{dNPR}[0, \mathrm{P}]$ and $\mathrm{dNPR}[1, \mathrm{P}]$ represent the level of noise respectively in the first and the second detection channel of the Principal detector, while dNPR $[0, S]$ and dNPR $[1, S]$ indicate the level of noise respectively in the first and second detection channel of the Spare detector. dNPR $[0, \mathrm{P}]$. dNPR $[1, \mathrm{P}]$, dNPR $[0, \mathrm{~S}]$ and dNPR $[1, \mathrm{~S}]$ are sampled every $500 \mathrm{~ms}$.

Detector switching occurs anytime one of the following conditions is met:

1. if $(\mathrm{dNPR}[0, \mathrm{P}]>\mathrm{T}\|\mathrm{dNPR}[1, \mathrm{P}]>\mathrm{T}\|(\mathrm{dNPR}[0, \mathrm{P}]+$ dNPR $[1, \mathrm{P}])>3 \mathrm{~T} / 2)$ AND $((\mathrm{dNPR}[0, \mathrm{P}]+\mathrm{dNPR}[1, \mathrm{P}])$ $>(\mathrm{dNPR}[0, \mathrm{~S}]+\mathrm{dNPR}[1, \mathrm{~S}]))$;

2. if one of the detection channels of the Principal detector is invalid while both channels are valid in the Spare detector;

3. if both Principal detector channels are invalid while at least one detection channel of the Spare detector is valid.

When a switch is performed, an event can be erroneously detected twice or missed. In order to prevent double events during detectors switching, all same events fired less than $200 \mathrm{~ms}$ apart will be ignored, including QRS-detections. In order to prevent missed events during a detector switch, the Spare detector maintains a memory of $200 \mathrm{~ms}$ of past events and fires them when it becomes Principal detector. Detector switching is suspended for one minute anytime a detector switch occurs.

\section{Results}

\subsection{Detector switching}

Several standard ECG databases [2] are available for the evaluation of beat detection algorithms. Tests on these well-annotated and validated databases provide reproducible and comparable results. Furthermore, these databases contain a large number of selected signals representative for the large variety of ECGs as well as signals that are rarely observed but clinically important. Unfortunately these databases are not suitable to test the goal achieved by the algorithm proposed in this paper, as it requires the availability of at least 8 channels. These databases contain only half-hour two channels ECG excerpts; they do not contain a particular noise due to lead fail or electrode contact noise.

In order to test our algorithm, a database was appositely created. This database contains 101 one-hour 12-lead Holter records accurately selected from 24-hour recording with a commercial analysis system (H-Scribe
TM, Mortata Inc.) on the basis of the amount of artifact. The records are chosen so that different kind of noise (High or Low Frequency) on various leads is considered. Manual annotation is made using WAVE [3].

\subsection{Validation of the algorithm}

According to [4], two parameters Sensitivity (Se) and Positive Predictivity $(+\mathrm{P})$ should be used to evaluate any beat detection algorithm; these parameters are defined by the following expressions:

$$
\mathrm{Se}=\frac{\mathrm{TP}}{\mathrm{TP}+\mathrm{FN}} \quad \text { and } \quad+\mathrm{P}=\frac{\mathrm{TP}}{\mathrm{TP}+\mathrm{FP}}
$$

where TP denotes the number of true positive detections, FN the number of false negatives, and FP the number of false positives. These two parameters are then calculated for the QRS (Q) and the ventricular arrhythmias (V). Beat by beat comparison is performed according to [4].

\subsection{Variable length observation window}

Any time the conditions in 2.3 are verified, detector switching is performed. When the signal on detector $\mathrm{P}$ is worse than the signal on detector $\mathrm{S}$ only for a short period of time, switching results in a flaw of the algorithm performance. Therefore, any time the conditions for a switch are met, a time interval of variable length (w) indicated as "observation window with variable length", is considered. To perform the switching the conditions should be met for the entire time interval $\mathrm{w}$.

$\mathrm{T}$ varied from 0 to 32 which corresponds to noise level equals to $0 \%$ and $50 \%$ respectively; w varied from 0 to 28 which are equivalent to a variable window length from $500 \mathrm{~ms}$ to $14.5 \mathrm{~s}$ (sampling time equals to $500 \mathrm{~ms}$ ).

The proposed algorithm (auto) was compared with the original single detector with fixed original channels 1,2 and 6 (default) and the modified independent leads with maximum amplitude (No-Switch) . The results can be seen in Table 4.

Table 4. Performance of algorithm.

\begin{tabular}{|l|c|c|c|}
\hline Indicator & Default & No-Switch & Auto, w=5 s \\
\hline QSe & 98.61 & 99.75 & 99.71 \\
\hline $\mathrm{Q}+\mathrm{P}$ & 98.45 & 99.31 & 99.29 \\
\hline $\mathrm{VSe}$ & 88.33 & 90.44 & 91.93 \\
\hline $\mathrm{V}+\mathrm{P}$ & 55.4 & 64.4 & 57.9 \\
\hline
\end{tabular}

No significant changes in the auto-algorithm performance were seen when only the $\mathrm{T}$ value was modified.

Figure 1 shows the behaviour of the auto-algorithm for the parameters $\mathrm{Q}$ Se, $\mathrm{Q}+\mathrm{P}, \mathrm{V}$ Se and $\mathrm{V}+\mathrm{P}$ in function of $\mathrm{w}$; any single point in the figures corresponds to the average value assumed by each of these parameters for the 33 values of T. From this figure, it is possible to observe that increasing the length of the observation 

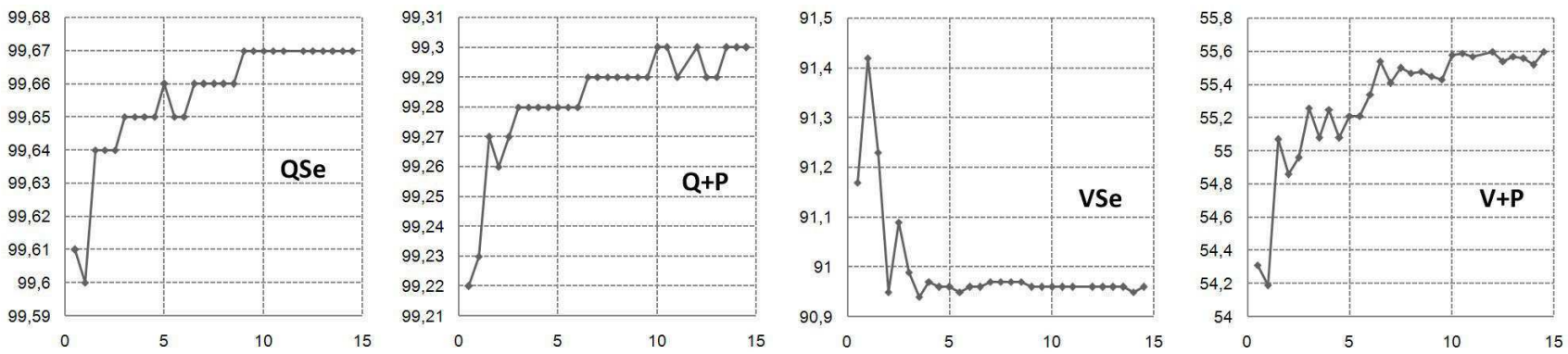

Figure 1. Variation of QRS sensitivity (QSe) and QRS positive predictivity (Q+P), ventricular arrhythmia sensitivity (VSe) and ventricular arrhythmia positive predictivity $(\mathrm{V}+\mathrm{P})$ of the automatic algorithm in $\%$ as a function of window length in $\mathrm{s}$.

window the parameters $\mathrm{Q}$ Se, $\mathrm{Q}+\mathrm{P}$ and $\mathrm{V}+\mathrm{P}$ increase. On the contrary V Se decreases.

\section{Discussion and conclusions}

This paper presents a new approach to automatic beat detection in the presence of a significant level of noise on some ECG leads: two identical detectors are running simultaneously and dynamically switching among them according to the level of noise on the leads used by the detectors is performed. The performance of the algorithm was tested on a database appositely created; this database contains 101 1-hour 12-lead Holter records selected from 24-hour recordings with a commercial analysis system on the basis of the amount of artifact. The performance of the algorithm was tested for various values of threshold for the estimation of the level of noise in a detection channel and for several values of the length of the observation window.

It can be concluded that the improvement of performance of the algorithm was caused mainly by the automatic choice of the best leads for detection based on mutual independence and QRS-amplitude. Dynamic switching of detector leads on the basis of the used noise indicators did not result in significant improvement.
Further investigation to better real time noise indicators might lead to better performance of the lead switching algorithm.

\section{References}

[1] Köhler B, Hennig C, Orglmeister R. The principles of software QRS detection. IEEE Engineering in Medicine and Biology 2002;1:42-58.

[2] Moody GB, Mark RG. The MIT-BIT3 arrhythmia database on CD-ROM and software for use with it. Computers in Cardiology. IEEE Computer Society Press 1990;17:185188.

[3] Software Wave Form Database, PhysioBank. Available: http://www.physionet.org/pn3/mghdb/

[4] ANSI/AAMI EC57: Testing and reporting performance results of cardiac rhythm and ST segment measurement algorithms (AAMI Recommended Practice/American National Standard), 1998. Available: http://www.aami.org

Address for correspondence

Cristiana Corsi, $\mathrm{PhD}$

D.E.I.S., University of Bologna

Via Venezia 52, 47023 Cesena - Italy

E-mail: cristiana.corsi3@unibo.it 